\title{
Road risk assessment using fuzzy Context-free Grammar based Association Rule Miner
}

\author{
S SARANYADEVI ${ }^{1, *} \mathbb{D}$, R MURUGESWARI $^{1}$ and S BATHRINATH $^{2}$ \\ ${ }^{1}$ Department of Computer Science and Engineering, Kalasalingam Academy of Research and Education, \\ Krishnankoil 626126, India \\ ${ }^{2}$ Faculty of Mechanical Engineering, Kalasalingam Academy of Research and Education, Krishnankoil 626126, \\ India \\ e-mail: saranyadevism@gmail.com
}

MS received 12 January 2019; revised 30 March 2019; accepted 16 April 2019; published online 27 May 2019

\begin{abstract}
Road traffic accidents are a major social concern as well as a crucial issue for the public in recent days due to the risk factors involved. Analysing and identifying the major risk factors of road accident is still a challenging task. In this paper, a fuzzy Context-free Grammar (FCFG)-based association rule mining (ARM) technique is proposed to categorize a heterogeneous road accident dataset into two categories based on the critical factors such as total number of accidents (TA), persons killed (PK) and persons injured (PI). The role of the fuzzy grammar in this paper is to govern the entire algorithm using the prescribed grammar rules to proceed further. The considered road accident dataset does not have class labels; hence there is a need to assign class labels for the available data instance. The accident data with assigned class labels are given as input to $K$-nearest neighbour (KNN) machine learning algorithm in order to train the classifier for testing purpose. Further, the collected test data from the user are utilized by the KNN classifier for carrying out the performance analysis of the proposed algorithm. The case study is conducted on the National Highway roads, India, to examine the proposed approach. The experimentations are executed for road accident records using MATLAB software and the analysis is made using the following performance measures: accuracy, recall or sensitivity, precision or specificity and $F 1$ score. A comparative study is accomplished with existing algorithms in order to show that the proposed algorithm works with improved accuracy of more than $83 \%$. The results suggested that the road users are responsible for the acceptance or rejection of safe or un-safe roads, respectively.
\end{abstract}

Keywords. Road accidents; data mining; association rule mining; formal grammars; $K$-nearest neighbour; fuzzy logic.

\section{Introduction}

Road traffic accidents are more frequent in India than other countries globally [1]. A report from National Crime Records Bureau (NCRB) stated that more than 1,35,000 traffic crash-related deaths take place annually in India. The rate of road accidents decreased by $4.1 \%$ in 2016 but fatalities rose by $3.2 \%$. In 2017, the percentage of roadrelated deaths was $3 \%$ or 4560 , which is lesser than that of the previous year's 1.51 lakh [2]. Analysis of road accidents using a number of records will reveal causes for the same and ensure that safety measures are taken, thereby reducing the number of accidents. For this, researchers were forced to use some effective techniques like data mining (DM), a machine learning technique.

DM is a sequence of processes to extract hidden, untouched data from a huge volume of data. Classification,

*For correspondence association and clustering are various DM concepts to cull out needed data from the entire corpus. An important data extraction technique, association rule mining (ARM), has been used in most research works to analyse road accident data combined with other DM techniques such as clustering and classification. An incremental sequential pattern mining approach was proposed to stay away from rescanning the complete database. Authors have used the depth first method and dynamic look ahead pruning scheme to eliminate infrequent patterns [3].

A $K$-mode clustering technique was used to segment the whole data and then ARM was applied to spot the situations that cause the accident [4]. In [5] the authors performed ARM with clustering based on the accident locations. Also, classification is used to analyse road traffic accidents with variations. Classification algorithms identify risk factors and the dependences among them based on severity levels [6].

Accidents are possible in any circumstances as they are unpredictable and hence accident-related data are inexact. 
Fuzzy logic seems to be an appropriate way to deal with uncertain data; hence this uncertainty enables us to use rule mining based on fuzzy logic, called fuzzy rule mining (FRM) in this research. A fuzzy logic modelling was proposed in [7] to find the key issues related to the environment of road network. A study on traffic crash location identification using fuzzy logic was discussed [8] to analyse and map out the exact crash locations. A model based on fuzzy rule [9] was developed to represent accident duration on expressways and to govern uncertain, complex data in the proposed accident monitoring system with improved accuracy. A weighted fuzzy ARM technique was proposed [10] based on information gain ratio to classify the medical data for disease identification. In FRM, users need to perform some elementary steps to enter all the details of input attributes and membership partitions and then to create fuzzy inference rules. A study on death distribution due to road traffic accidents was performed pertaining to road safety to know the most contributing factors to road accident deaths [11]. The study provided details about the fatal rates based on road segments, expressways, bends and junction points, etc.

ARM can be performed through formal grammar [12, 13] - a key concept of Formal Language Theory [14]. Formal grammar is often used in programming languages to get the required output. Context-free Grammar (CFG) is one of the grammars in Formal Language Theory where a set of rules appear recursively, resulting in output data. A CFG-based ARM or pattern mining was performed in $[15,16]$ based on genetic algorithm by limiting the search space for extracting the interested association rules or patterns. The proposed model uses a fuzzy Context-free Grammar (FCFG), an extension of CFG, which was used to tackle data's unclear, unpredictable and imprecise nature. In this paper, a novel model is developed by combining FRM and CFG, which is termed as FCFG ARM (Fuzzy Context-free Grammar based Association Rule Mining).

The paper is organized as follows. Section 2 describes the problem definition and methodology of the proposed algorithm, including ARM, fuzzy logic, FCFG and $K$ nearest neighbour (KNN) algorithm. Section 3 explains about the functionality of proposed FCFG-based ARM algorithm with detailed steps and process flow. Section 4 presents a detailed explanation of the dataset identified for the experiments and demonstrates the analysis of FCFGbased categorization of results, testing the dataset with different combinations based on KNN, performance evaluation using confusion matrix (CM) and accuracy comparison to existing algorithms. Section 5 contains the concluding remarks and the future scope of research.

\section{Problem definition and methodology used}

The proposed work presents an innovative self-learning approach of automatically performing a series of steps such as assigning the input of fuzzy partition limits based on mean value of all the available attributes of the dataset. Based on the fuzzy inputs with partitions, fuzzy rules are created and then the fuzzy output is produced without any human involvement. However, generally, in FRM, user has to give all information of input attributes for the assignment and creation of fuzzy membership values and fuzzy rules respectively. To date, there is no other research work carried so far using formal grammars with combination of FRM for performing road accident studies.

This paper analyses real-time data of road accident for 2015. We focus only on the National Highway across India and mined accident-prone areas. Based on this we conclude the preferences of choosing safe or unsafe roads, thereby reducing road accidents. This section contains an overview of ARM, fuzzy logic and KNN methods used in the proposed algorithm.

\subsection{Overview of ARM, fuzzy logic and FCFG}

2.1a ARM: ARM concept has been applied to study road crash data to understand the correlation between two characteristics involved in road accident occurrence. An association rule of the form $A \rightarrow B$ implies that if $A$ occurs then $B$ will also occur. $A$ and $B$ are two different itemsets. Consider that there are $n$ number of transactions in dataset D where each transaction $T \in D$. Let a set of items be $I=\left\{I_{1}, I_{2}, \ldots, I_{n}\right\}$. An itemset $A$ or $B$ will occur in $T$ if and only if $A$ or $B \subseteq T$. The association rule $A \rightarrow B$ provides that $A \subset I, B \subset I$ and $A \cap B=\phi$. Association rules can be built using two main interestingness measures: support and confidence $[17,18]$.

2.1b Fuzzy logic: Fuzzy logic can be suitably applied to represent road accident data and human-related factors [19]. Most common real-world data are numeric or alphanumeric values. Hence there is a necessity to convert such data into fuzzy acceptables. This process is known as fuzzification, which starts by finding the unique values of individual variables or attributes to map the values in the discourse within the scale of $[0,1]$. The next step defines fuzzy inference rules for each variable defined in the first step to produce a fuzzy output. Finally, defuzzification is used to revert fuzzy output values to their original crisp value.

2.1c FCFG: FCFG can be defined using the base form, CFG [20].

A CFG quadruple $G=(\Sigma, N, P, S)$, where

- $\Sigma$ is the finite set of terminal symbols;

- $N$ a finite set of non-terminal symbols;

- $P$ a finite set of production rules of the form

$$
A \rightarrow \alpha, A \in N, \alpha \in(\Sigma \cup N)^{*} .
$$

- $S \in N$ is the starting symbol.

A FCFG $G_{F}$ is an extension of CFG, where production rule $\mathrm{P}$ is of the form 


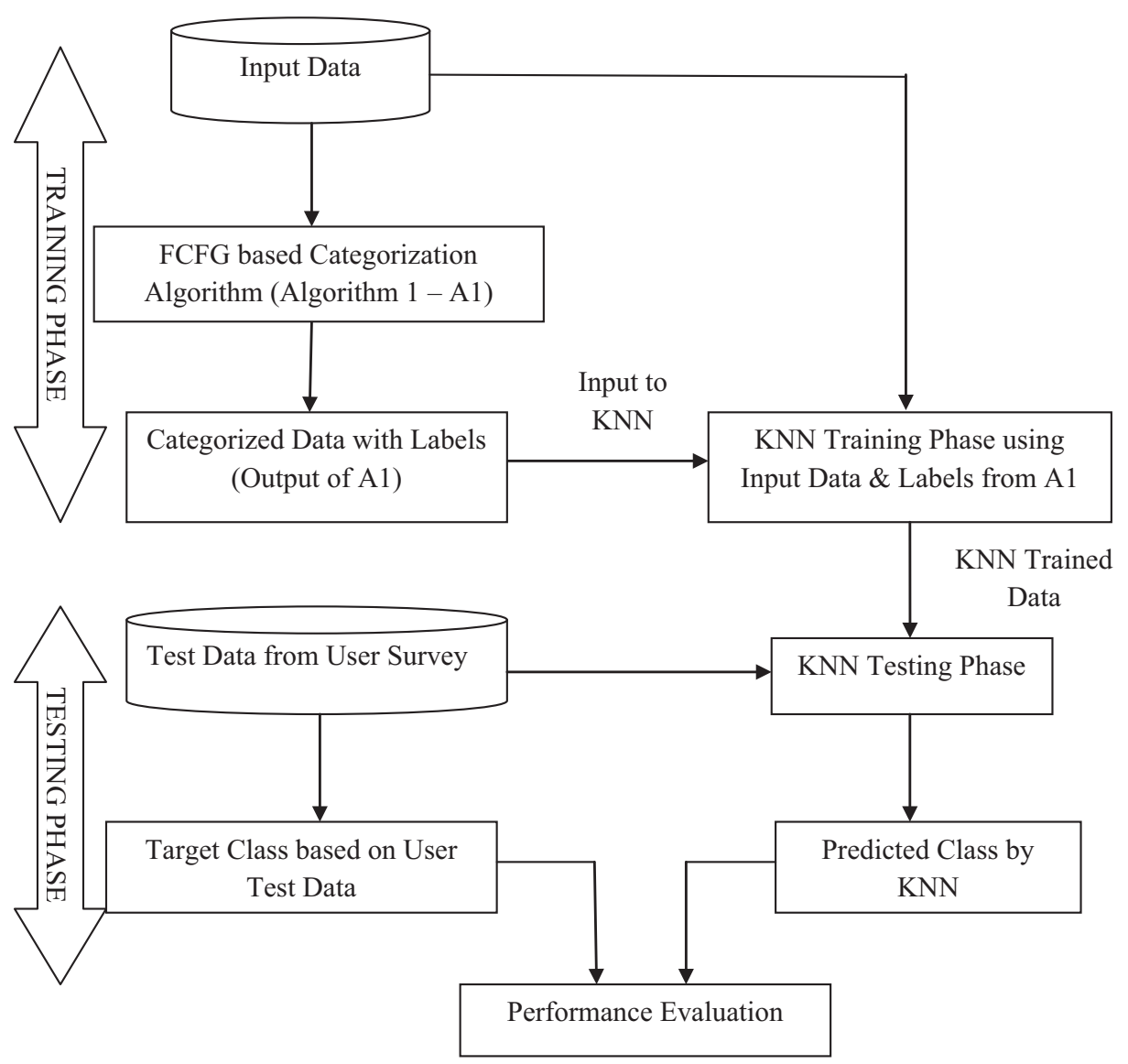

Figure 1. Process flow of FCFG-based ARM algorithm.

$$
A \stackrel{\mu}{\longrightarrow} \alpha, A \in N, \alpha \in(\Sigma \cup N)^{*}, \mu \in[0,1] .
$$

Here $\mu$ is called the membership function. $S \in N$ is the starting symbol. More specifically, the elements of $\mathrm{P}$ are of the form

$$
\mu(A \rightarrow \alpha)=\rho, \rho>0
$$

where $\alpha$ and $\rho$ are strings in $(\Sigma \cup N)^{*}$ and $\rho$ is the grade of membership of $\alpha$ and $\rho$.

FCFG in DM: DM is applicable in almost all types of applications. DM is a series of processes with the potential to discover knowledge from large databases. DM enables implementation of Formal Language Theory as an additional feature to customize existing methods or algorithms to produce enhanced results. Formal Language Theory uses formal grammar to provide definitions of programming languages and build a structure of compilers used in computational linguistics and is a study of human language applied to pattern recognition, mathematical logic, DM, etc. To date, there are various studies and approaches proposed on formal grammars as mentioned in literature; however, this research applies an FRM technique using formal grammars, specifically FCFG.

2.1d $K N N$ : A well-known machine learning algorithm that is widely used to classify any supervised data is the $\mathrm{KNN}$ algorithm. KNN algorithm used for regression and classification is a non-parametric learning algorithm [21]. KNN can perform classification for an unknown and unclassified database. Many distance measures are used to calculate the distance between two examples as a form of attribute in a dataset.

\section{Proposed FCFG-based ARM algorithm}

The proposed FCFG-based ARM algorithm is divided into two main phases: training phase and testing phase. Figure 1 is a pictorial representation of proposed algorithm's process flow.

From figure 1, the fuzzy ARM is used for training purpose and the KNN algorithm is used for testing purpose. The steps are as follows. 
Step 1: First the user provides input data to the processing system based on fuzzy grammar. Since the road accident data are unlabelled, the proposed algorithm correctly determines the class labels for any unknown data. Based on the proposed approach, the system performs all the steps based on grammar rules (as explained in sections 3.1a-3.1c) without human involvement and produces the categorized output with class labels. Data (with labels) obtained from this step are named as training data.

Step 2: Then these data are given as input to the machine learning algorithm (say KNN) to validate the results of the proposed method (training the KNN classifier).

Step 3: The next step is KNN testing phase, in which the test data are taken from user survey with the class labels called as target class by user.

Step 4: The KNN algorithm will also produce some output class labels called as predicted class.

Step 5: Based on the target classes by the user and the predicted classes by the KNN algorithm, the performance of proposed method is evaluated.

\subsection{Training phase}

The training phase has three steps: (i) preprocessing, (ii) creating fuzzy rules and (iii) FCFG-based result categorization and testing phase. First, in the preprocessing step, the upper, medium and lower limits are defined from fuzzy membership variables for all the attributes present. Next the fuzzy rules are generated to run the fuzzy processor in order to obtain the fuzzy output after evaluation of rules. Then by using fuzzy output, the results are categorized into two main categories, safe and unsafe roads, which are named as categorized output labels.

3.1a Preprocessing (fuzzification): Preprocessing step is a very important process of defining the fuzzy variables of all the available attributes of given dataset. Defining fuzzy membership values for the input parameters is known as the fuzzification step. The proposed algorithm is an enhancement of ARM with fuzzy grammar.

This research work uses a triangular membership function as it is effortless and reduces the error rate in most research [22, 23]. The triangular membership function needs three input range values such as low, medium and high. After identification of unique values, the algorithm assigns the membership values $[0,1]$, and membership functions of various attributes given in the input data follow the generation of an output membership function.

Let the number of road accident attributes (RA) be $n$, i.e., $R A_{i}=A_{1}, A_{2}, \ldots, A_{n}$, where $i=1,2, \ldots, n$ and the number of road accident instances (RI) in the dataset be $m$, i.e., $R I_{j}=I_{1}, I_{2}, \ldots, I_{m}$, where $j=1,2, \ldots, m$. First, the grammar finds the equivalent values for low, medium and high fuzzy limits for all the attributes of dataset by calculating the mean value of the attributes. The mean value is calculated using Eq. (4):

$$
\text { Mean }_{R A}=\frac{\text { Sum of all values of attribute }}{\text { Total number of instances }} .
$$

For any attribute $A_{i}$, the mean is obtained from Eq. (4) and can be written as

$$
\operatorname{Mean}_{R A}=\frac{\operatorname{Sum}\left(A_{i}\right)}{\operatorname{Length}\left(I_{j}\right)} .
$$

With the help of mean value of a specific attribute, the lower, medium and higher limits can be set as follows:

- If the attribute value is higher than the mean value then the corresponding fuzzy value can be considered as 1 or high.

- If the attribute value is lower than the mean value then the corresponding fuzzy value can be considered as 0 or low.

- If the attribute values is equal or nearest to the mean value then the corresponding fuzzy value is considered as 0.5 or medium.

For any attribute with available instance of the dataset, these points can be shown as

$$
V_{F}=\left\{\begin{array}{ccc}
\text { High } & : & V_{A t t}>V_{A_{i}} \\
\text { Medium } & : & V_{A t t}=V_{A_{i}} \\
\text { Low } & : & V_{A t t}<V_{A_{i}}
\end{array}\right.
$$

where

$$
\begin{aligned}
V_{F} & =\text { Fuzzy Membership value, } V_{A t t} \\
& =\text { Value of Attributes and } \\
V_{A_{i}}= & \text { Mean value of each } i:(1 \leq i \leq n)
\end{aligned}
$$

The conditions in Eq. (6) are considered by the grammar rules to develop the fuzzy processing system. In the normal fuzzy process, all the membership values with partition limits (low, medium, high, etc.) are set manually for all the available attributes of dataset; however, in the proposed FCFG ARM, the following steps are carried out automatically:

i. Finding mean and assigning fuzzy values using Eqs. (4)-(6).

ii. Creating fuzzy rules (section $3.1 b$ ) based on the membership value, $V_{F}$.

iii. Obtaining the fuzzy output generated by fuzzy inference rules.

iv. Performing categorization (section 3.1c) based on fuzzy output obtained in step iii.

3.1b Creating fuzzy rules (fuzzy rule inference system): Fuzzy rules or conditions are created using the fuzzy membership variables defined in the preprocessing step and the fuzzy rules are evaluated using the fuzzy processor. Fuzzy outputs are generated based on the conditions of low, medium and high 
values of each attribute defined in fuzzy membership step. Defuzzification is the process of producing output decisions based on the membership functions defined.

3.1c FCFG based result categorization: The fuzzy processing system provides output by analysing the given fuzzy rules. Based on the fuzzy output the categorization of data is performed. Initially the first output data are placed in first category. Then the proposed algorithm compares one output instance to all other instances that are produced by fuzzy processor, to place data in specific categories. Finally, the difference between the output membership functions of two subsequent instances is considered to assign them in the same category if difference is less; otherwise, a subsequent category is chosen. This process is repeated till the entire fuzzy output data are placed into all the available categories. The categories obtained from this step are termed as labels (classes). The whole process of categorization by means of output labels is mentioned in Algorithm 1.

\section{Algorithm 1: FCFG-based categorization algorithm (training phase)}

Initialization: Instances: $I_{1}, I_{2}, \ldots, I_{n}$; Categories: $C_{1}, C_{2}, \ldots, C_{m} ;$ Attributes: $A_{1}, A_{2}, \ldots, A_{k}$

Input: uncategorized training dataset $=\operatorname{DRoad}_{\text {Train }}$,

number of instances $=n$, number of attributes $=k$, number of categories $=m$,

Fuzzy Rule (FR), Rules to assign fuzzy input (RU), Upper Limit (UL), Lower Limit

(LL), Medium Limit (ML), Fuzzy Output (FOP), Number of Categories (NC).

\section{Algorithm:}

1. Preprocessing (Finding Upper and lower limits)

2. for $(i=1 ; j<k ; i++)$ begin

3. for $(j=1 ; j<n ; j++)$ begin

4. $U L=\operatorname{Maximum}\left(A_{i}\left(I_{j}\right)\right)$

5. $\quad L L=\operatorname{Minimum}\left(A_{i}\left(I_{j}\right)\right)$

end

6. $\quad M L=\operatorname{Avg}(U L, L L)$

end

7. Fuzzy Rule Inference System (Setting Fuzzy Rule)

8. $\quad$ set $l=L L, u=U L$ and $M L$

9. for $(i=1 ; i<n ; i++)$ begin

10. $\quad F O P(i)=A(i)$

end

11. FCFG based Categorization

12. set $C_{1}=I_{1}$

13. for $(i=1 ; i<$ length $(F O P) ; i++)$ begin

14. for $(j=1 ; j<k ; j++)$ begin

15. for $(r=1 ; r<m ; r++)$ begin

16. $\quad P(F O P)=F O P((i))$

17. $\quad$ if $(P(F O P)=F O P((i)))$

18. $\quad C_{1} \leftarrow I_{i+1} \quad$ //assign to same category

19. else

20. $C_{m+r} \leftarrow I_{i+1} \quad$ //assign to the next categories end for end for

end for

21. repeat steps 5 and 6 until $r<m$

22. repeat steps 5 and 6 until $i<\operatorname{length}(F O P)$

23. Print the categorized data, DRoad ${ }_{\mathrm{Clv}} \quad / /$ Training Data with Class labels

Output: Categorized data with labels, DRoad $_{\mathrm{Clv}}$ 


\subsection{Testing phase}

In testing phase, the test data are considered to know whether the algorithm has produced the correct categorization results or not. The fuzzy processor produces output labels for all the instances of the dataset, and now these labels need to be tested with the identified test data to judge the performance of proposed algorithm. Algorithm 2 presents the steps of testing the algorithm using machine learning technique (say KNN in this study) for unknown class labels. Generally, for any machine learning algorithm, the user needs to provide all the input and class label details manually; however, using the proposed technique, the machine learning algorithm works automatically (using unlabelled data as a labelled one), by its own as a self-learning method. users based on road accident dataset 2016 as testing data (mentioned in the Appendix section).

\subsection{Road accident dataset}

Road accident dataset [24] is used for experiments in this research. The dataset was collated from reports of the central government road transport and highways ministry's Transport Research Wing (TRW). The report includes recent and long-term trends, normalized indicators for road accidents, injuries and fatalities, details from the top 13 states in terms of road accidents, persons killed, persons injured, severity of road accidents, accidents in terms of classification of roads, urban and rural road accidents,

Algorithm 2: Testing with machine learning (KNN) algorithm

Input: Instances: $I_{1}, I_{2}, \ldots, I_{n} ;$ Categories: $C_{1}, C_{2}, \ldots, C_{m}$; Attributes: $A_{1}, A_{2}, \ldots, A_{k}$

Uncategorized testing dataset $=$ DRoadUser $_{\text {Test }}$, class value $\left(\right.$ DRoad $\left._{\text {Clv }}\right)$, target class $(T C)$ based

on DRoadUser ${ }_{\text {Test }}$, Output Class from KNN algorithm = OPC.

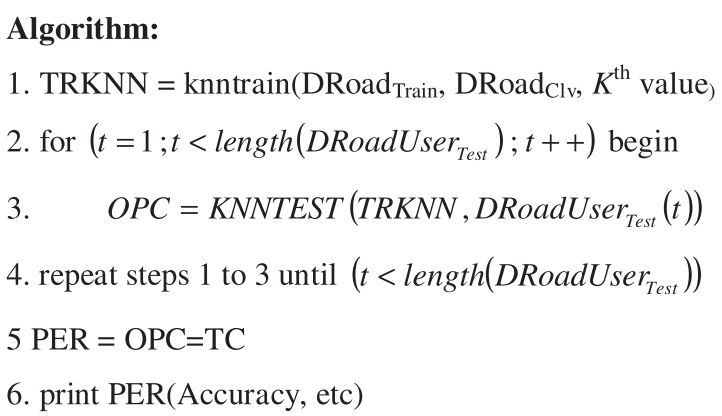

Output: Categorized output data, PER // Tested data after comparison with performance metrics.

To the best of our knowledge no research was done on the study of road accident data using formal grammars. This research work develops a processing system based on fuzzy grammar to model road accident data with independent variables, and categorizes unknown data by considering three significant variables total number of accidents (TA), persons killed (PK) and persons injured (PI).

\section{Results and discussion}

The experimentations are executed using an Intel Core-i5 processor, with 8-GB RAM and a 32-bit Operating System, and are implemented using MATLAB software. In this research, road accident dataset for the year 2015 is considered as the training data and the survey results from accidents based on vehicle type, time of occurrence, victim's age and gender, and reason for road accidents. Data on 8062 accidents were used for this experiment. Table 1 gives a brief outline of the attributes used in the dataset for the proposed algorithm.

\subsection{Analysis of categorization}

The purpose of categorizing a heterogeneous dataset is to reduce data analysis complexity for research or survey. The proposed model classifies the whole data into two categories, $\mathrm{C} 1$ and $\mathrm{C} 2$ for unsafe and safe roads, respectively.

Category 1 (C1): Unsafe roads based on the analysis of three independent attributes, namely TA, PK and PI in different National Highway segments of 37 states in India. 
Table 1. Attribute descriptions of road accident data (for National Highways).

\begin{tabular}{|c|c|c|c|c|}
\hline & $\begin{array}{l}\text { S1. } \\
\text { no. }\end{array}$ & $\begin{array}{l}\text { Attribute } \\
\text { name }\end{array}$ & Values & Description \\
\hline \multirow[t]{7}{*}{ Input } & 1 & $\begin{array}{l}\text { Type of } \\
\text { vehicles }\end{array}$ & $\begin{array}{c}\text { Two Wheelers } \\
\text { Auto Rickshaws } \\
\text { Cars, Jeeps, } \\
\text { Taxis } \\
\text { Bus } \\
\text { Trucks, Tempos, } \\
\text { MAVs } \\
\text { Other Motor } \\
\text { Vehicles } \\
\text { Others }\end{array}$ & $\begin{array}{c}\text { Type of } \\
\text { vehicles used }\end{array}$ \\
\hline & 2 & Person's age & $\begin{array}{c}18-24 \\
25-34 \\
35-44 \\
45 \text { and above } \\
\text { Age not known }\end{array}$ & $\begin{array}{c}\text { Age of persons } \\
\text { killed }\end{array}$ \\
\hline & 3 & $\begin{array}{l}\text { Person's } \\
\text { gender }\end{array}$ & $\begin{array}{c}\text { Male } \\
\text { Female }\end{array}$ & $\begin{array}{c}\text { Gender of } \\
\text { persons } \\
\text { killed }\end{array}$ \\
\hline & 4 & $\begin{array}{l}\text { Weather } \\
\text { conditions }\end{array}$ & $\begin{array}{l}\text { Fine } \\
\text { Mist/fog } \\
\text { Cloudy } \\
\text { Rainy } \\
\text { Other } \\
\text { extraordinary } \\
\text { weather } \\
\text { conditions }\end{array}$ & $\begin{array}{c}\text { Type of weather } \\
\text { conditions }\end{array}$ \\
\hline & 5 & $\begin{array}{l}\text { Time of } \\
\text { accident } \\
\text { occurrence }\end{array}$ & $\begin{array}{c}6-9.00 \mathrm{~h} \\
18-21.00 \mathrm{~h}\end{array}$ & $\begin{array}{l}\text { Time of } \\
\text { accident } \\
\text { occurrence } \\
\text { (6-9 am and } \\
6-9 \text { pm) }\end{array}$ \\
\hline & 6 & Location & $\begin{array}{c}\text { Near } \\
\text { school/college } \\
\text { Near } \\
\text { bazaar/market } \\
\text { Near hospital } \\
\text { Near residential } \\
\text { area }\end{array}$ & $\begin{array}{c}\text { Location where } \\
\text { the accident } \\
\text { occurred }\end{array}$ \\
\hline & 7 & $\begin{array}{l}\text { Nature of } \\
\text { accident }\end{array}$ & $\begin{array}{l}\text { Overturning } \\
\text { Head-on } \\
\text { collision } \\
\text { Hit and run } \\
\text { Safe road } \\
\text { Un-safe road }\end{array}$ & $\begin{array}{l}\text { Type of } \\
\text { accident }\end{array}$ \\
\hline
\end{tabular}

C1 includes 1863 of 8062, implying that 1863 roads are not safe for use.

Category 2 (C2): Safe roads based on the analysis of three independent attributes namely, TA, PK and PI in different National Highway segments of 37 states in India. C2 consists of 6199 of 8062 data, implying that 6199 roads are safe for use.

\subsection{Testing road accident dataset}

The dataset can be tested based on KNN algorithm. This section has two parts. In the first, the road accident dataset (2015) already categorized by the proposed algorithm is tested. In the second part, a new road accident dataset (2016) is tested based on 2015 training data.

4.3a Testing with categorized dataset (2015): From section 4.2 we obtained two categories: safe and unsafe class of roads from the road accident dataset 2015 using the proposed algorithm. Now we test the same data using KNN algorithm to compare accuracy of results from both algorithms. We received 100\% accuracy for known and categorized data.

4.3b Testing with un-categorized dataset (2016): Here we test the new road accident dataset 2016, which has the same format with unknown classes, using the KNN algorithm. The results reveal more than $83 \%$ accuracy in categorizing unknown real-time data, which is shown in figure 2 .

\subsection{Testing using human survey}

The outputs are based on road accident dataset 2015, as a training data using the proposed algorithm. The KNN algorithm was used to check accuracy of results from the algorithm. To confirm the proposed work, we surveyed 50 people of different age groups. This survey was undertaken on randomly selected 50 data from the 2016 road accident dataset. The data of 50 focused on 3 independent variables: TA, PK and PI. The data handed over to people as a questionnaire to list their preferences on using safe or unsafe roads are given in table 2. People gave the data to check road conditions based on accident occurrence and its results. After obtaining these results from the people, we noticed that human-selected preferences are the same as the machine-learned preferences with more than $83 \%$ of

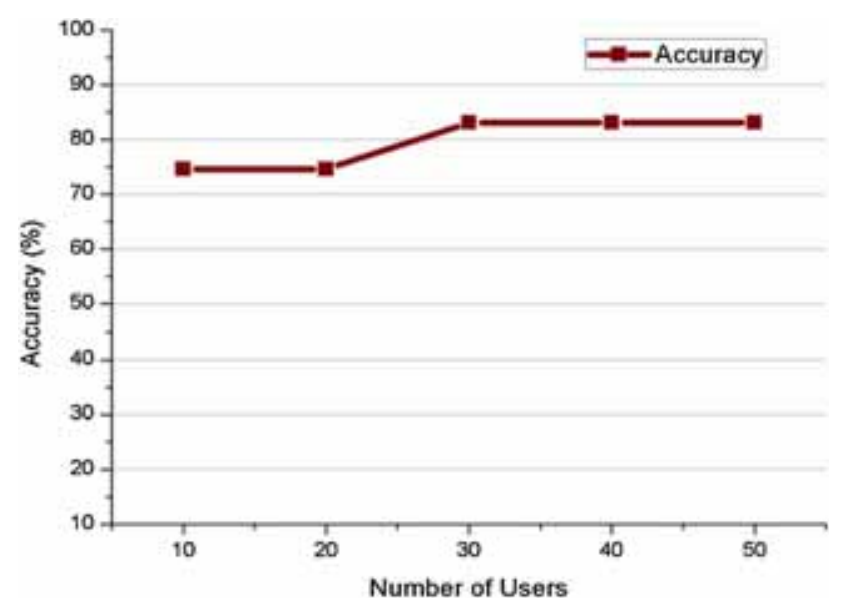

Figure 2. Categorization accuracy for 50 users. 
Table 2. Sample data for human survey.

\begin{tabular}{|c|c|c|c|c|c|c|c|c|}
\hline Sl. no. & Total no. of accidents & Persons killed & Persons injured & User1 & User2 & User3 & $\ldots$ & User50 \\
\hline 1 & 36020 & 11493 & 41673 & 0 & 1 & 0 & $\ldots$. & 1 \\
\hline 2 & 64764 & 11241 & 63033 & 1 & 1 & 0 & $\ldots$ & 1 \\
\hline 3 & 14937 & 4020 & 13421 & 1 & 0 & 0 & $\ldots$ & 1 \\
\hline 4 & 2946 & 387 & 2716 & 1 & 1 & 1 & $\ldots$ & 1 \\
\hline 5 & 10240 & 5714 & 6148 & 0 & 1 & 1 & $\ldots$ & 0 \\
\hline
\end{tabular}

$* 0$ - unsafe road; 1 - safe road.

accuracy. Thus the proposed algorithm works better in any dataset type: either real-time or existing benchmark datasets.

\subsection{Performance evaluation using CM}

The accuracy of proposed algorithm is demonstrated with the help of a CM. The matrix consists of two classes: actual and predicted class. The row and column of the matrix represent actual and predicted classes, respectively. The values True Positive (TP) and True Negative (TN) are the degree of correctly classified classes as target classes, and False Positive (FP) and False Negative (FN) are the degree of wrongly classified classes as target classes.

The CMs have been obtained individually for 10, 20, 30, 40 and 50 users and the corresponding accuracy values are $74.6 \%, 74.6 \%, 83.1 \%, 83.1 \%$ and $83.1 \%$, respectively, which are displayed in table 3 .

A sample of 50 users is considered for evaluating the performance of proposed method in terms of accuracy, and is shown in figure 2. From figure 2 it is clearly observed that the accuracy varies in the range of 70-85 for individual users. Initially the accuracy starts at $74 \%$ for 10 users; it gradually increases when the number of users increases and finally it is maintained constantly at the value of around $83 \%$, for 50 users. If the number of users increases, the accuracy of the proposed model will also be increased. From table 3, the accuracy of the proposed algorithm is maintained at the same value, $83.1 \%$, from users 30 till 50 .

Similarly some other measures such as precision, recall and $F$-measure have also been calculated from $\mathrm{CM}$. Precision $(P)=\mathrm{TP} /(\mathrm{TP}+\mathrm{FP})$, recall $(R)=\mathrm{TP} /(\mathrm{TP}+\mathrm{FN})$ and $F$-Measure $(F)=(2 R P) /(R+P)$. Using these formulas, the obtained values for precision, recall and $F$-measure are $0.783,1$ and 0.8264 , respectively, for 50 users.

In order to handle the data imbalance, other performance metrics such as recall or sensitivity, precision or specificity and $F 1$ score are also discussed. Since the categorization results produced by the proposed algorithm are not balanced for training data (road accident dataset 2015), (i.e., out of 8062 input records, category C1 includes 1863 (unsafe) and category C2 includes 6199 (safe) records), the identified metrics are considered additionally in this analysis.
Table 3. Confusion matrix (CM).

\begin{tabular}{|c|c|c|c|c|c|}
\hline \multicolumn{4}{|c|}{ Predicted class } & \multicolumn{2}{|c|}{ Predicted class } \\
\hline \multicolumn{3}{|c|}{ (a) $\mathrm{CM}$ for 10 users. } & \multicolumn{3}{|c|}{ (b) CM for 20 users. } \\
\hline \multirow[t]{5}{*}{ Actual class } & $\mathrm{TP}$ & FP & Actual class & $\mathrm{TP}$ & FP \\
\hline & 31 & 13 & & 32 & 14 \\
\hline & $\mathrm{FN}$ & $\mathrm{TN}$ & & $\mathrm{FN}$ & $\mathrm{TN}$ \\
\hline & 0 & 13 & & 1 & 12 \\
\hline & $\begin{array}{r}\text { Accur } \\
\text { use }\end{array}$ & $\begin{array}{l}\text { for } 10 \\
74.6 \%\end{array}$ & & $\begin{array}{r}\text { Accu } \\
\text { use }\end{array}$ & $\begin{array}{l}\text { for } 20 \\
74.6 \%\end{array}$ \\
\hline \multicolumn{3}{|c|}{ (c) $\mathrm{CM}$ for 30 users. } & (d) $\mathrm{CM}$ & for 40 & \\
\hline \multirow[t]{5}{*}{ Actual class } & $\mathrm{TP}$ & FP & Actual class & $\mathrm{TP}$ & FP \\
\hline & 36 & 10 & & 36 & 10 \\
\hline & $\mathrm{FN}$ & $\mathrm{TN}$ & & FN & $\mathrm{TN}$ \\
\hline & 0 & 13 & & 0 & 13 \\
\hline & $\begin{array}{r}\text { Accur } \\
\text { use }\end{array}$ & $\begin{array}{l}\text { for } 30 \\
83.1 \%\end{array}$ & & $\begin{array}{r}\text { Accu } \\
\text { use }\end{array}$ & $\begin{array}{l}\text { for } 40 \\
83.1 \%\end{array}$ \\
\hline \multicolumn{6}{|c|}{ (e) $\mathrm{CM}$ for 50 users. } \\
\hline \multirow[t]{5}{*}{ Actual class } & $\mathrm{TP}$ & FP & & & \\
\hline & 36 & 10 & & & \\
\hline & $\mathrm{FN}$ & $\mathrm{TN}$ & & & \\
\hline & 0 & 13 & & & \\
\hline & $\begin{array}{r}\text { Accur } \\
\text { use }\end{array}$ & $\begin{array}{l}\text { for } 50 \\
83.1 \%\end{array}$ & & & \\
\hline
\end{tabular}

Table 4. Survey results for 50 users.

\begin{tabular}{lcc}
\hline Number of users & C1 (safe) & C2 (un-safe) \\
\hline 10 & 4 & 6 \\
20 & 9 & 11 \\
30 & 13 & 17 \\
40 & 18 & 22 \\
50 & 22 & 28 \\
\hline
\end{tabular}

For test data (road accident dataset 2016 - the test data consist of 60 records), the survey results produced by 50 $(10,20,30,40,50)$ users placed in two categories are displayed in table 4 .

4.5a Recall or sensitivity: Recall is a performance measure of how many chosen instances are appropriate. Figure 3 presents in graphical format the performance of $\mathrm{KNN}$ classifier with recall values for 50 users. From figure 3, the recall value lies between 0.73 and 0.91 . 


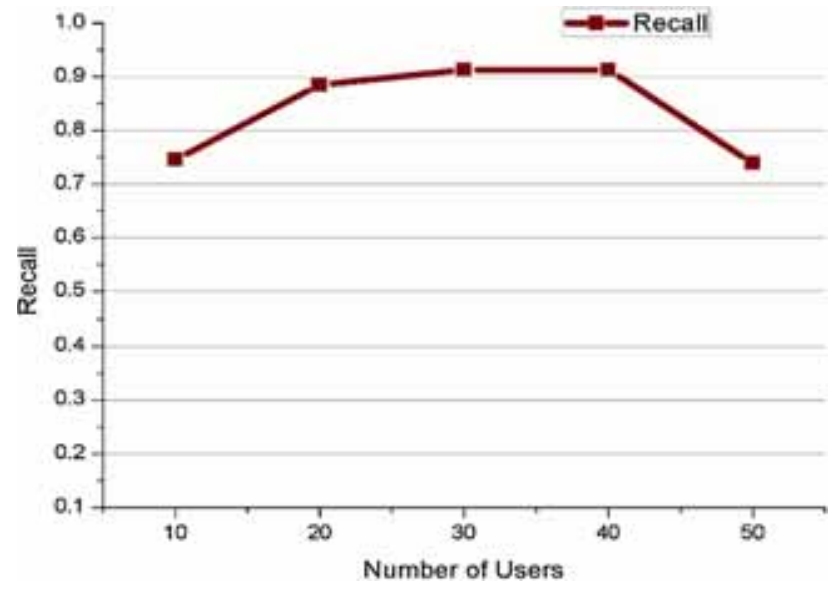

Figure 3. Representation of recall or sensitivity for 50 users.

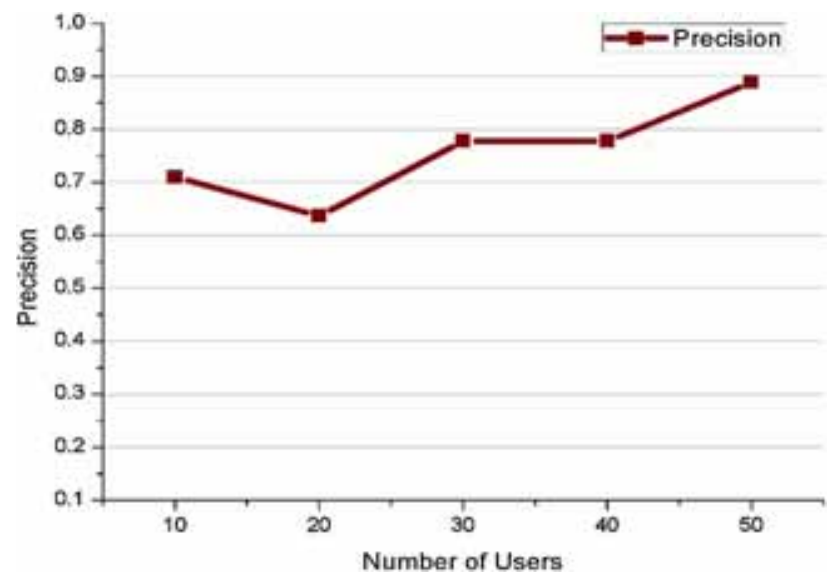

Figure 4. Representation of precision or specificity for 50 users.

4.5b Precision or Specificity: Precision is performance measure of how many appropriate instances are chosen. Figure 4 represents the graphical format of the performance of KNN classifier with precision values for 50 users. From figure 4 , the precision value lies between $0.63-0.88$.

4.5c F1 score: $F 1$ score is a performance measure that indicates the weighted mean of precision and recall. Figure 5 presents $F 1$ score values in graphical format for 50 users. From figure 5, the value lies between 0.38 and 0.72 .

4.5d Accuracy comparison with existing algorithms: Figure 6 is the graphical representation of accuracy comparison of the proposed FCFG-based ARM method with various existing algorithms. It is evidently illustrated that the proposed algorithm has more accuracy than the existing ones. Table 5 presents various methods or algorithms used in existing work with accuracy value.

\subsection{Synoptic view of data analysis}

This study focuses on issues that lead to road accidents by considering a few attributes from the main dataset [24]

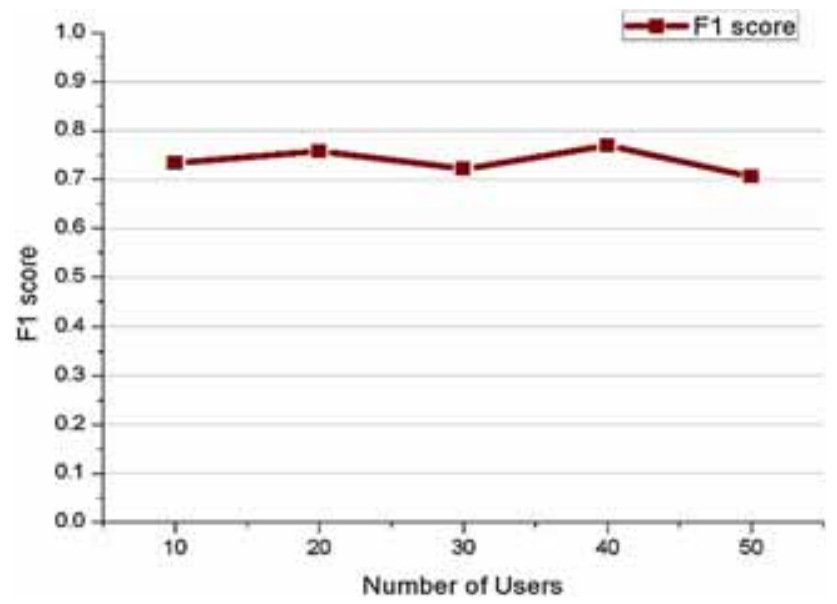

Figure 5. Representation of $F 1$ score for 50 users.

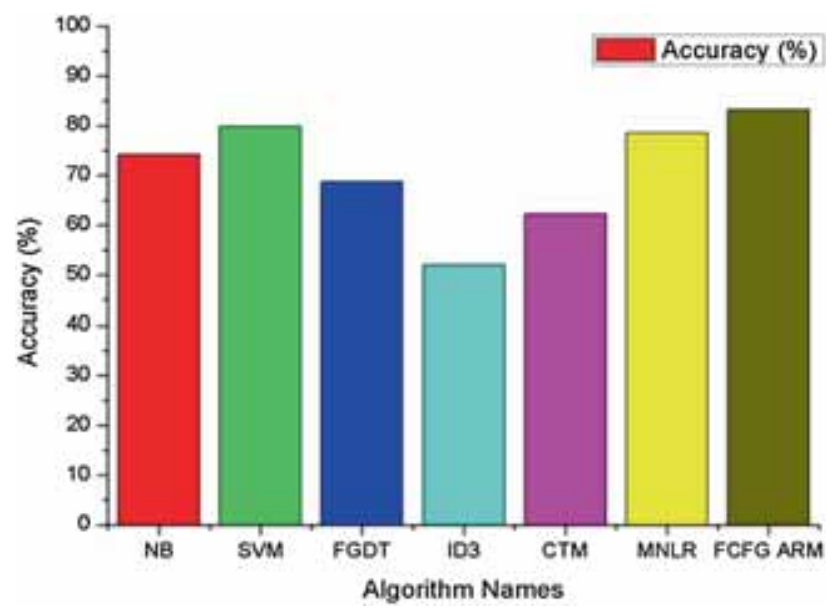

Figure 6. Comparison of accuracy with existing algorithms.

Table 5. Accuracy comparison.

\begin{tabular}{lcc}
\hline Reference & Algorithm name & $\begin{array}{c}\text { Accuracy } \\
(\%)\end{array}$ \\
\hline$[25]$ & Naive Bayes (NB) & 74.14 \\
& Support Vector Machine (SVM) & 79.79 \\
{$[26]$} & Fuzzy Granular Decision Tree (FGDT) & 68.7 \\
& Fuzzy ID3 & 52 \\
{$[21]$} & Classification Tree Modelling (CTM) & 62.2 \\
{$[27]$} & Multinomial Logistic Regression - & 78.57 \\
& classification (MNLR) \\
& FCFG ARM (Proposed) & $\mathbf{8 3 . 1}$ \\
\hline
\end{tabular}

pertaining to this research. There are totally 8062 records of National Highway road accidents from India's 36 states for the year 2015, used in this research. The proposed algorithm successfully categorized 8062 records into two different categories: safe (6199) and unsafe (1863). 
Categorization is done using different variables based on attributes like type of vehicle, age of person, gender, weather conditions, time of accident, type of location, nature of accident, TA, PK and PI. The variables of each attribute can be referred to from table 1. Most accidents occurred between 6 am and 9 am (Day) as compared with 6 pm and 9 pm (Night).

\section{Conclusion}

In this paper, a FCFG is used to build an ARM algorithm that is more efficient than the elementary FRM. The important part of the proposed algorithm is self-learning, which enables it to categorize a dataset without anterior information. In the elementary FRM, each step such as assignment of $V_{\mathrm{F}}$ (fuzzy membership value) for each attribute and creation of fuzzy rules were carried out manually, that is, the user must enter all the inputs. However, in the proposed FCFG ARM, every task is selflearned by the algorithm itself to give meaningful results; the only task of user is to upload the required dataset. The performance analysis is done using the metrics accuracy, recall or sensitivity, precision or specificity and $F 1$ score. Comparison between existing algorithms and proposed FCFG-based ARM algorithm is performed; the proposed algorithm is proved to be better than the existing ones in terms of accuracy.

This output will provide decision makers with sufficient knowledge to reach exact and intensified decisions to evade hazardous route and create awareness of such places by providing recommendations to improve road safety.

\section{Acknowledgement}

The first author would like to thank the management of Kalasalingam Academy of Research and Education (KARE) for providing fellowship to carry the research work.

\section{Appendix}

Sample training data (2015). 


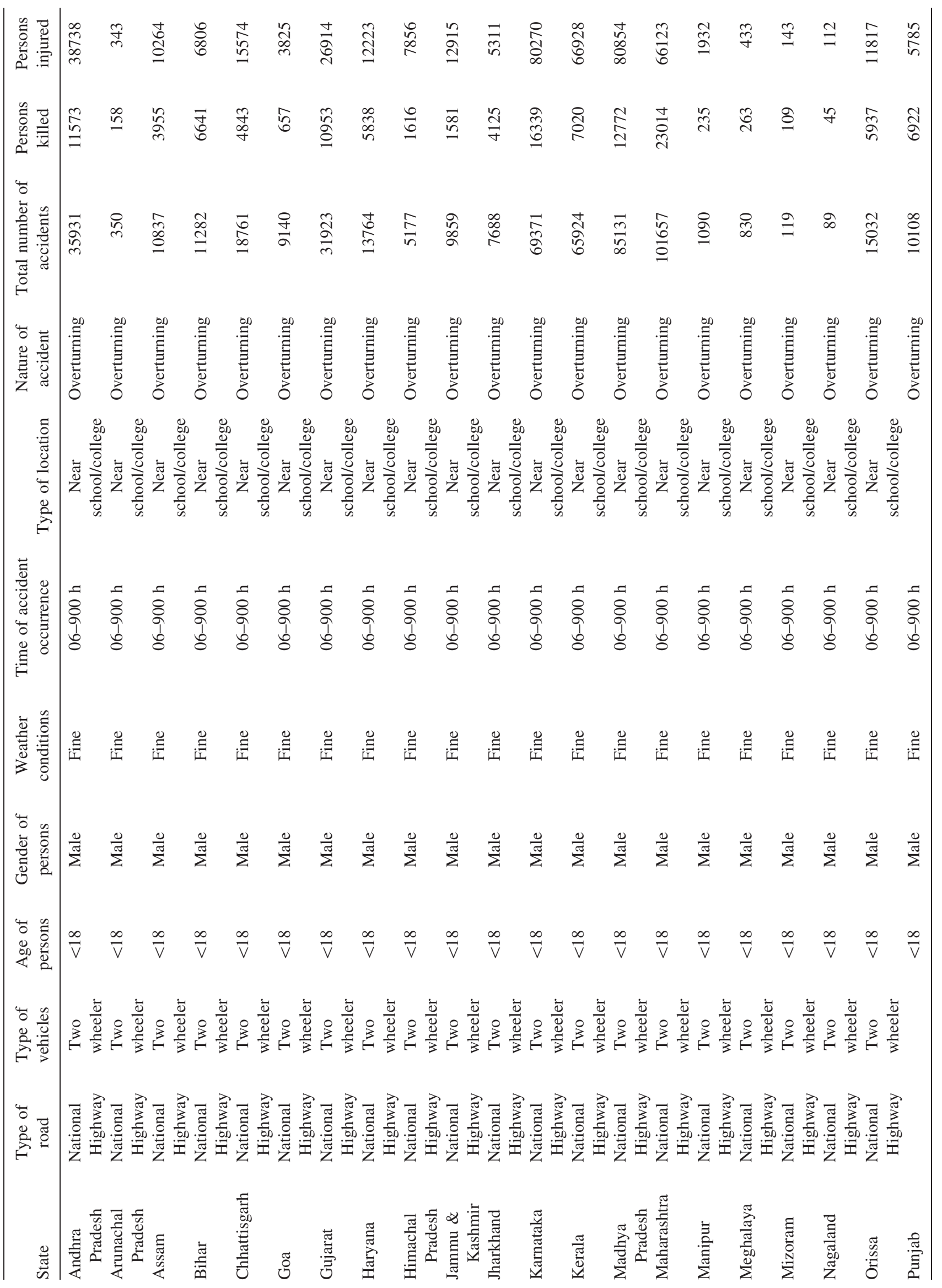




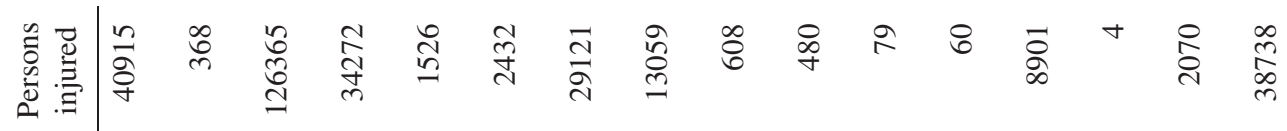

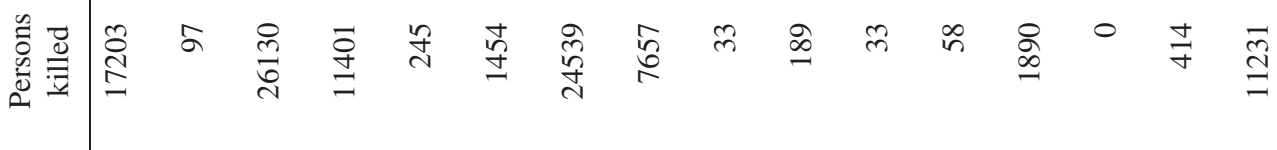

4

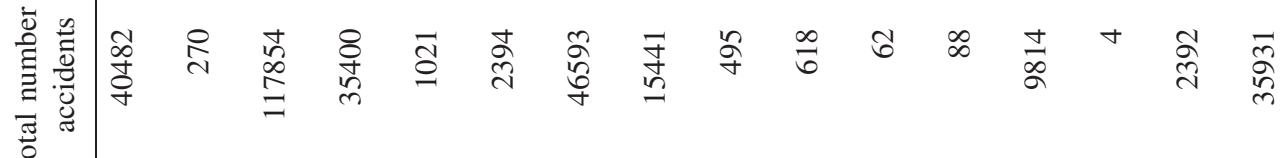

$\stackrel{\text { }}{\ominus}$

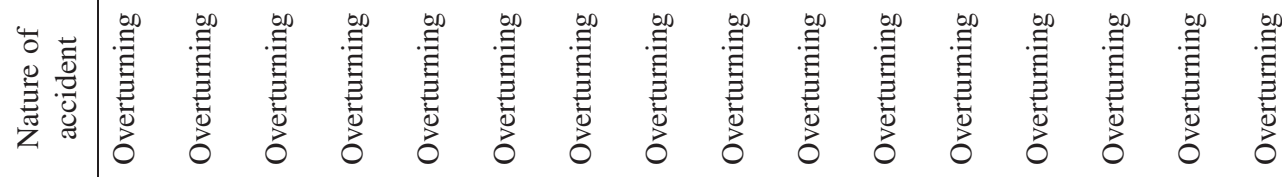

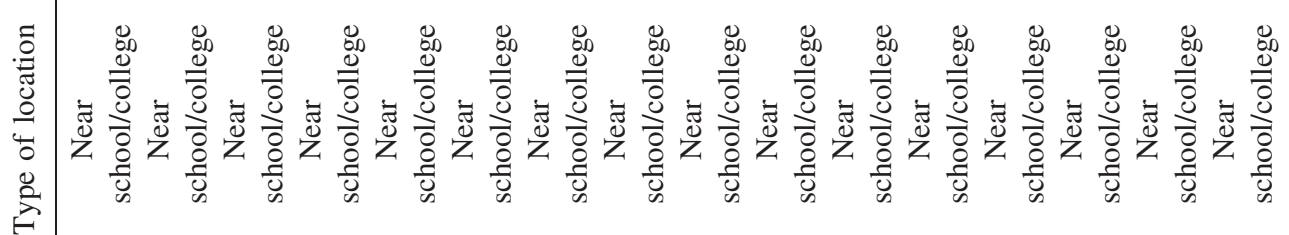

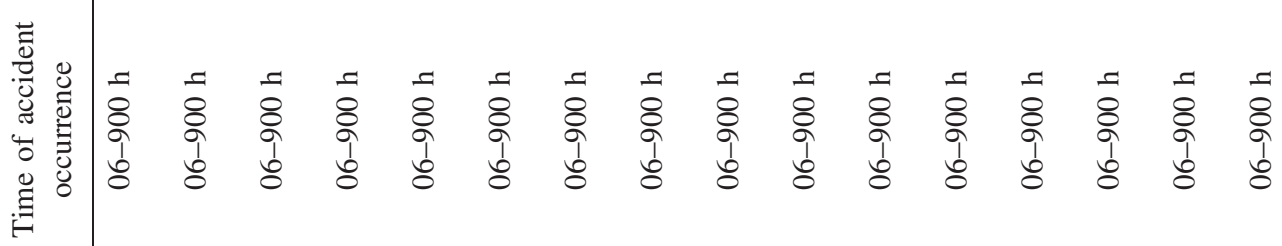

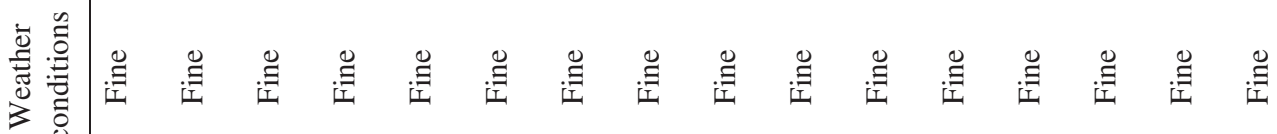

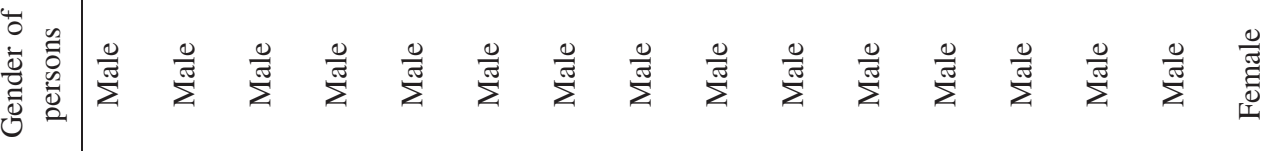

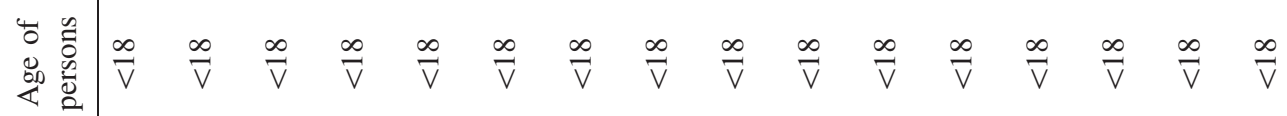

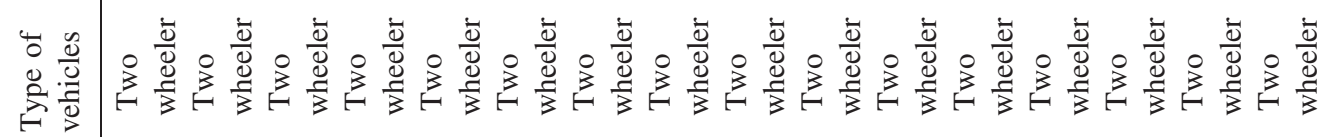

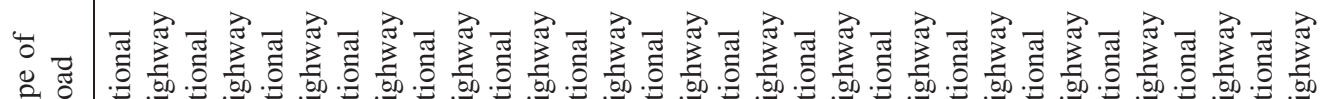

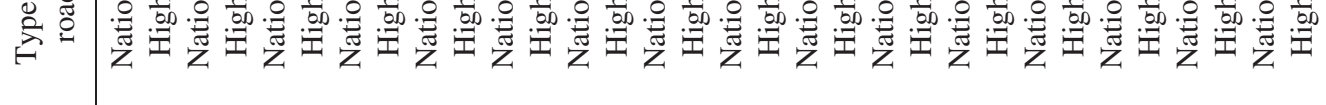

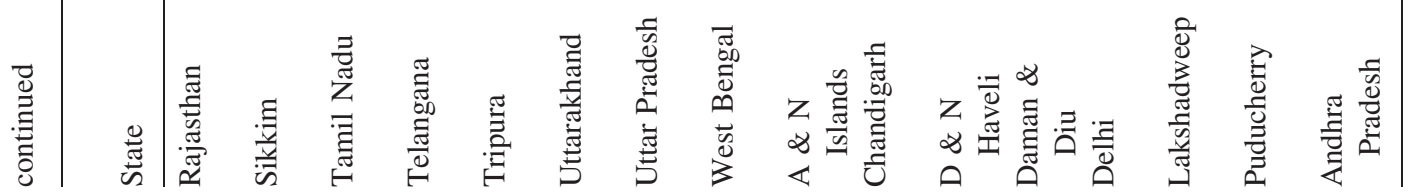


Sample testing data (2016).

\begin{tabular}{|c|c|c|}
\hline Total no. of accidents & Persons killed & Persons injured \\
\hline 36020 & 11493 & 41673 \\
\hline 64764 & 11241 & 63033 \\
\hline 14937 & 4020 & 13421 \\
\hline 2946 & 387 & 2716 \\
\hline 10240 & 5714 & 6148 \\
\hline 16042 & 6425 & 15244 \\
\hline 370 & 235 & 408 \\
\hline 8778 & 3279 & 6660 \\
\hline 8658 & 5724 & 5003 \\
\hline 7166 & 4149 & 4594 \\
\hline 72330 & 17303 & 76945 \\
\hline 70031 & 7109 & 68508 \\
\hline 88538 & 14010 & 82925 \\
\hline 570 & 195 & 367 \\
\hline 53 & 32 & 81 \\
\hline 87 & 38 & 87 \\
\hline 6877 & 1425 & 5568 \\
\hline 420 & 233 & 566 \\
\hline 11986 & 3941 & 8468 \\
\hline 9627 & 5479 & 5933 \\
\hline 18227 & 4259 & 15450 \\
\hline 34794 & 12825 & 27488 \\
\hline 645 & 92 & 1124 \\
\hline 594 & 166 & 307 \\
\hline 81 & 87 & 44 \\
\hline 8993 & 4211 & 8849 \\
\hline 5347 & 4003 & 3012 \\
\hline 14119 & 8469 & 13417 \\
\hline 172 & 80 & 184 \\
\hline 54030 & 14560 & 56978 \\
\hline 2828 & 895 & 4167 \\
\hline 3772 & 626 & 4337 \\
\hline 6319 & 3451 & 4183 \\
\hline 488 & 174 & 728 \\
\hline 1649 & 1077 & 1519 \\
\hline 31837 & 18683 & 19269 \\
\hline 4040 & 2146 & 2571 \\
\hline 33055 & 7433 & 36468 \\
\hline 34143 & 3773 & 36661 \\
\hline 70 & 50 & 108 \\
\hline 39 & 16 & 49 \\
\hline 3656 & 828 & 2638 \\
\hline 162 & 60 & 95 \\
\hline 2139 & 329 & 2158 \\
\hline 525 & 154 & 274 \\
\hline 69 & 59 & 48 \\
\hline 327 & 56 & 139 \\
\hline 2261 & 1542 & 2119 \\
\hline 37555 & 26464 & 27745 \\
\hline 12301 & 4712 & 7139 \\
\hline 235 & 22 & 233 \\
\hline 229 & 124 & 121 \\
\hline 54 & 39 & 79 \\
\hline 65 & 30 & 38 \\
\hline 21587 & 8364 & 20751 \\
\hline 229 & 153 & 432 \\
\hline
\end{tabular}

continued

\begin{tabular}{lcc}
\hline Total no. of accidents & Persons killed & Persons injured \\
\hline 615 & 210 & 862 \\
1575 & 1038 & 1327 \\
31999 & 18547 & 19193 \\
\hline
\end{tabular}

\section{References}

[1] Traffic accidents in India. https://en.wikipedia.org/wiki/Traf fic_collisions_in_India

[2] Road Accident Deaths 2018 https://timesofindia.indiatimes. com/india/2017-saw-3-decline-in-road-accident-deaths/arti cleshow/62828118.cms

[3] Boghey R K and Singh S 2016 A sequential tree approach for incremental sequential pattern mining. Sädhanā 41(12): $1369-1380$

[4] Kumar S and Toshniwal D 2015 A data mining framework to analyze road accident data. J. Big Data 2(1): 26

[5] Kumar S and Toshniwal D 2016 A data mining approach to characterize road accident locations. J. Mod. Transp. 24(1): $62-72$

[6] Kwon O H, Rhee W and Yoon Y 2015 Application of classification algorithms for analysis of road safety risk factor dependencies. Accid. Anal. Prev. 75: 1-15

[7] Driss M, Benabdeli K, Saint-Gerand T and Hamadouche M A 2015 Traffic safety prediction model for identifying spatial degrees of exposure to the risk of road accidents based on fuzzy logic approach. Geocarto Int. 30(3): 243-257

[8] Imprialou M I M, Quddus M and Pitfield D E 2014 High accuracy crash mapping using fuzzy logic. Transp. Res. Part C Emerg. Technol. 42: 107-120

[9] Wang A, An N, Chen G, Li L and Alterovitz G 2015 Accelerating wrapper-based feature selection with K-nearestneighbor. Knowl. Based Syst. 83: 81-91

[10] Nithya N S and Duraiswamy K 2014 Gain ratio based fuzzy weighted association rule mining classifier for medical diagnostic interface. Sadhana 39(1): 39-52

[11] Darma Y, Karim M R and Abdullah S 2017 An analysis of Malaysia road traffic death distribution by road environment. Sādhanā 42(9): 1605-1615

[12] Luna J M, Romero J R and Ventura S 2012 Design and behavior study of a grammar-guided genetic programming algorithm for mining association rules. Knowl. Inf. Syst. 32(1): 53-76

[13] Luna J M, Romero J R and Ventura S 2013 Grammar-based multi-objective algorithms for mining association rules. Data Knowl. Eng. 86: 19-37

[14] Hahn K S 1989 Investigation of a fuzzy grammar for automated visual inspection. Doctoral dissertation, Texas Tech University

[15] Ventura S and Luna J M 2016 Pattern mining with evolutionary algorithms. Berlin: Springer, pp. 1-190

[16] Ventura S and Luna J M 2018 Supervised descriptive pattern mining. Springer International Publishing, https://doi.org/10. 1007/978-3-319-98140-6

[17] Das S, Dutta A, Avelar R, Dixon K, Sun X and Jalayer M 2018 Supervised association rules mining on pedestrian 
crashes in urban areas: identifying patterns for appropriate countermeasures. Int. J. Urban Sci. 23(1): 30-48

[18] Kumar S and Toshniwal D 2015 Analysing road accident data using association rule mining. In: Proceedings of the 2015 International Conference on Computing, Communication and Security (ICCCS), IEEE, pp. 1-6

[19] Dimitriou L and Vlahogianni E I 2015 Fuzzy modeling of freeway accident duration with rainfall and traffic flow interactions. Anal. Methods Accid. Res. 5: 59-71

[20] Hopcroft J E 2008 Introduction to automata theory, languages, and computation. Pearson Education India

[21] Jung S, Qin X and Oh C 2016 Improving strategic policies for pedestrian safety enhancement using classification tree modeling. Transp. Res. Part A Policy Pract. 85: 53-64

[22] Selvi O 2009 Traffic accident predictions based on fuzzy logic approach for safer urban environments, case study: Izmir Metropolitan Area. $\mathrm{PhD}$ thesis
[23] Mandal S N, Choudhury J P and Chaudhuri S B 2012 In search of suitable fuzzy membership function in prediction of time series data. Int. J. Comput. Sci. Issues 9(3): 293-302

[24] Road Accidents Statistics in India 2015 http://pibphoto.nic. in/documents/rlink/2016/jun/p20166905.pdf

[25] Moghaddam H K and Wang X 2014 Vehicle accident severity rules mining using fuzzy granular decision tree. In: Proceedings of the International Conference on Rough Sets and Current Trends in Computing. Cham: Springer, pp. 280-287

[26] Pakgohar A, Tabrizi R S, Khalili M and Esmaeili A 2011 The role of human factor in incidence and severity of road crashes based on the CART and LR regression: a data mining approach. Procedia Comput. Sci. 3: 764-769

[27] Castro Y and Kim Y J 2016 Data mining on road safety: factor assessment on vehicle accidents using classification models. Int. J. Crashworth. 21(2): 104-111 